Sportwiss 2009 · 39:4-4

DOI 10.1007/s12662-009-0020-8

๑) Springer Medizin Verlag 2009

\author{
J. Fischer ${ }^{1} \cdot$ G. Doll-Tepper ${ }^{2}$ B B. Strauß ${ }^{3}$ \\ ${ }^{1}$ Bundesinstitut für Sportwissenschaft, Bonn \\ ${ }^{2}$ Deutscher Olympischer Sportbund, Frankfurt a. M. \\ ${ }^{3}$ Deutsche Vereinigung für Sportwissenschaft, Hamburg
}

\title{
Kontinuität und Innovation
}

Liebe Leserinnen und Leser,

mit der vorliegenden Ausgabe halten Sie das erste Heft des 39. Jahrgangs der Zeitschrift "Sportwissenschaft" in den Händen - das erste Heft der Zeitschrift, das im Springer-Verlag Heidelberg erscheint. Die (institutionellen) Herausgeber der "Sportwissenschaft" - das Bundesinstitut für Sportwissenschaft (BISp), der Deutsche Olympische Sportbund (DOSB) und die Deutsche Vereinigung für Sportwissenschaft (dvs) - freuen sich auf die neue Partnerschaft mit dem renommierten Wissenschaftsverlag und gehen von einem wichtigen Schub in der Entwicklung der Zeitschrift aus. Wir erhoffen uns mit dieser wegweisenden Verbindung, dass nicht nur die Zeitschrift, sondern auch die deutsche Sportwissenschaft an Profil und Sichtbarkeit gewinnt.

Dazu beitragen werden sicherlich die nun endlich vorhandene Onlinepräsenz der Zeitschrift (sportwissenschaft.springer.de) und die Einbindung in eine international aufgestellte Verlagsgruppe mit ihren vielfältigen professionellen Möglichkeiten.

Die Kontinuität in der inhaltlichen Arbeit ist durch das bisherige Herausgeberkollegium gesichert. Allen Kolleginnen und Kollegen, insbesondere Prof. Dr. Michael Krüger und Prof. Dr. Eike Emrich danken wir ganz herzlich für ihre Arbeit und wissen die Zeitschrift dort in guten Händen. Im Laufe des Jahres werden sich die drei institutionellen Herausgeber mit den beiden geschäftsführenden Herausgebern und dem Springer-Verlag treffen, um erste Erfahrungen auszutauschen und über weitere Maßnahmen zu beraten, wie die Zeitschrift sich zukünftig in einem neuen Umfeld erfolgreich platzieren kann.
Dieses neue Umfeld ist dadurch gekennzeichnet, dass die Zeitschrift für ihren Fortbestand zahlreiche Abonnenten, neue wie alte, benötigt. Wir hoffen auch hier auf eine positive Entwicklung, weil wir vom Potenzial dieser Zeitschrift überzeugt sind.

Ganz wesentlich wird der Erfolg der "Sportwissenschaft" davon abhängen, dass Sie, verehrte Leserinnen und Leser, auch die Rolle von Autorinnen und Autoren einnehmen und Beiträge über Ihre Forschungen in dieser Zeitschrift veröffentlichen. Diese Publikation ist ein Forum für alle sportwissenschaftlichen Teildisziplinen, von den naturwissenschaftlichen über die verhaltens- und sozialwissenschaftlichen bis hin zu den geisteswissenschaftlichen. Nutzen Sie die Möglichkeit, in diesem wichtigen Forum der deutschsprachigen Sportwissenschaft Ihre besten Arbeiten zu publizieren.

Für die Herausgeber:

Jürgen Fischer

Direktor des Bundesinstituts für Sportwissenschaft

Prof. Dr. Gudrun Doll-Tepper Vizepräsidentin Bildung und Olympische Erziehung des Deutschen Olympischen Sportbundes

Prof. Dr. Bernd Strauß Präsident der Deutschen Vereinigung für Sportwissenschaft

\section{Korrespondenzadresse}

Prof. Dr. B. Strauß

Institut für Sportwissenschaft, Universität Münster Horstmarer Landweg 62b, 49149 Münster bstrauss@uni-muenster.de 